\title{
Establishment of characterization system for testing multi silicon micro-strip sensors at the University of Delhi
}

\author{
Chakresh Jain ${ }^{1}$ \\ Centre for Detector and Related Software Technology, Department of Physics and Astrophysics, \\ University of Delhi, New Delhi, India \\ E-mail: interuniversechakresh15@gmail.com \\ Geetika Jain, Abhijeet, Ajay Kumar, Ashutosh Bhardwaj, Kirti Ranjan \\ Centre for Detector and Related Software Technology, Department of Physics and Astrophysics, \\ University of Delhi, New Delhi, India
}

\begin{abstract}
A major concern with the use of silicon sensor in nuclear and particle physics experiments is its survival in the intense radiation environment. The unprecedented increase in fluence in these experiments affects its long-term sustainability due to both bulk and surface damage, resulting in the deterioration of its static and dynamic properties. Hence, stringent tolerance criteria are imposed on these sensors to maintain the physics performance of an experiment. This necessitates the testing of the silicon sensors' performance under temperature and humidity controlled, dark and dust free, environment.

Our Group at the University of Delhi is in the process of establishing such a characterization system, for the first time in India, for testing a large array of silicon micro-strip sensors. A set of electrical characterization units, capable of providing $3000 \mathrm{~V}$ and measuring pico-Ampere currents and pico-Farad capacitances, are installed in the facility. Among other features, the probe station has a capability to translate in three directions, with a step size of 2 micro-meter over the range of $20 \mathrm{~cm}$ in XY directions. The entire system is interfaced through the Automated Characterization Suite (ACS) software and can be programmed in such a way that one does not need to intervene manually as it switches from one silicon strip to another. Several measurements involving currents, capacitances and resistances can be performed for the total, strip and inter-strip parameters. It is primarily envisioned to utilize the setup for the qualification of micro-strip silicon sensors for the CMS outer tracker in the high-luminosity LHC upgrade. In this work, we present the details of this state-of-the-art characterization system and measurements performed on silicon strip sensors.
\end{abstract}

The 27th International Workshop on Vertex Detectors - VERTEX2018

22-26 October 2018

MGM Beach Resorts, Muttukadu, Chennai, India

\footnotetext{
${ }^{1}$ Speaker

(c) Copyright owned by the author(s) under the terms of the Creative Commons

Attribution-NonCommercial-NoDerivatives 4.0 International License (CC BY-NC-ND 4.0).
} 


\section{Introduction}

Si sensors are placed nearest the interaction point of the CMS experiment, where they face the maximum shower of charged and uncharged particles produced because of the proton-proton collision at the LHC, CERN. This leads to severe radiation damage of surface and bulk of the detector material. Further, in the high luminosity LHC physics programme (HL-LHC) [1] the peak instantaneous luminosity will be increased by six times of the current level. To meet the desired physics goals of the CMS experiment, the CMS experiment will get the existing p-on-n Si detectors in the tracker replaced by more radiation hard n-on-p detectors in the CMS Phase II upgrade [2]. Due to the stringent technical requirements, these detectors are required to be extensively tested under the temperature and humidity controlled environment for their qualification. For this purpose, the University of Delhi has established a characterization system and proposed to test a fraction of multi silicon micro-strip sensors which would be installed in the outer tracker of CMS experiment in Phase II upgrade.

\section{Basic equipments for characterizing sensors and their specifications}

- A description of various instruments including electrical characterization units is provided below which are installed at DU for silicon micro-strip sensor characterization.

A) MPI Probe station : It is a light-tight dark chamber which is integrated on a vibration free table. Inside the chamber a nickel plated chuck is installed on which Device Under Test (DUT) can be placed and measurements can be done in a humidity and temperature controlled environment. There is an isolation ring in the chuck to isolate $2 \mathrm{kV}$ from ground. There is also a provision of vacuum holes on the chuck to hold the DUT firmly to avoid any warping and movement of sensor during the course of measurements. The chuck is having a XYZ translation stage which is capable of moving the chuck $210 / 340 \mathrm{~mm}$ in X/Y direction with a step size of 2 $\mu \mathrm{m}$ and $10 \mathrm{~mm}$ in $\mathrm{Z}$ direction with a step size of $1 \mu \mathrm{m}$. Further, it also has theta rotation of 5 degrees. The temperature of the chuck can be controlled by a temperature controller between $15^{\circ} \mathrm{C}$ to $200^{\circ} \mathrm{C}$. There are $4 \mathrm{RF}$ Micro-Positioners placed on probe platen with magnetic base. Also, there are 2 Micro-Positioners attached with the chuck platform to ensure the continued biasing of DUT during the chuck translation. Inside the probe station a microscope is mounted which is integrated with a video camera allowing one to view the enlarged view of micro-sized elements of the DUT on a touchscreen.

B) Set of electrical instruments : There are a number of electrical units which are used to characterize all the required parameters for the sensor. These include a single channel high voltage Source Measuring Unit (SMU), K2657A, which can source and measure voltages up to $3 \mathrm{kV}$ and can also source and measure currents from fA order to fraction of Ampere; a dual channel SMU, K2636B, which can source and measure voltages up to $200 \mathrm{~V}$ and also currents from fA order to tens of Ampere; a LCR meter, E4980A, which can measure capacitance from $1 \mathrm{e}-18$ to $1 \mathrm{e} 18 \mathrm{~F}$ and can source $\mathrm{AC}$ voltage from 0 to $2 \mathrm{~V}_{\mathrm{RMS}}$ with a frequency of $20 \mathrm{~Hz}$ to 2 $\mathrm{MHz}$ and can also source a DC voltage from 0 to $40 \mathrm{~V}$; an Isolation box to decouple the LCR 
meter from high voltages; a Low Interconnect Module, to provide a common LOW terminal to the SMUs; a Protection Module to protect instruments from high voltages; a switching matrix, with a semiconductor matrix card which can be operated at a maximum voltage of $200 \mathrm{~V}$ and a high voltage switching card which can be operated at a maximum voltage of $1.3 \mathrm{kV}$, which are used to switch between the connections for different measurements.

All these electrical instruments and probe station have been interfaced by Automated Characterization Suite (ACS) software through which we can send instructions to the electrical units and probe system depending on the measurement to be done. Figure 1 shows the complete characterization system along with the set of electrical instruments.

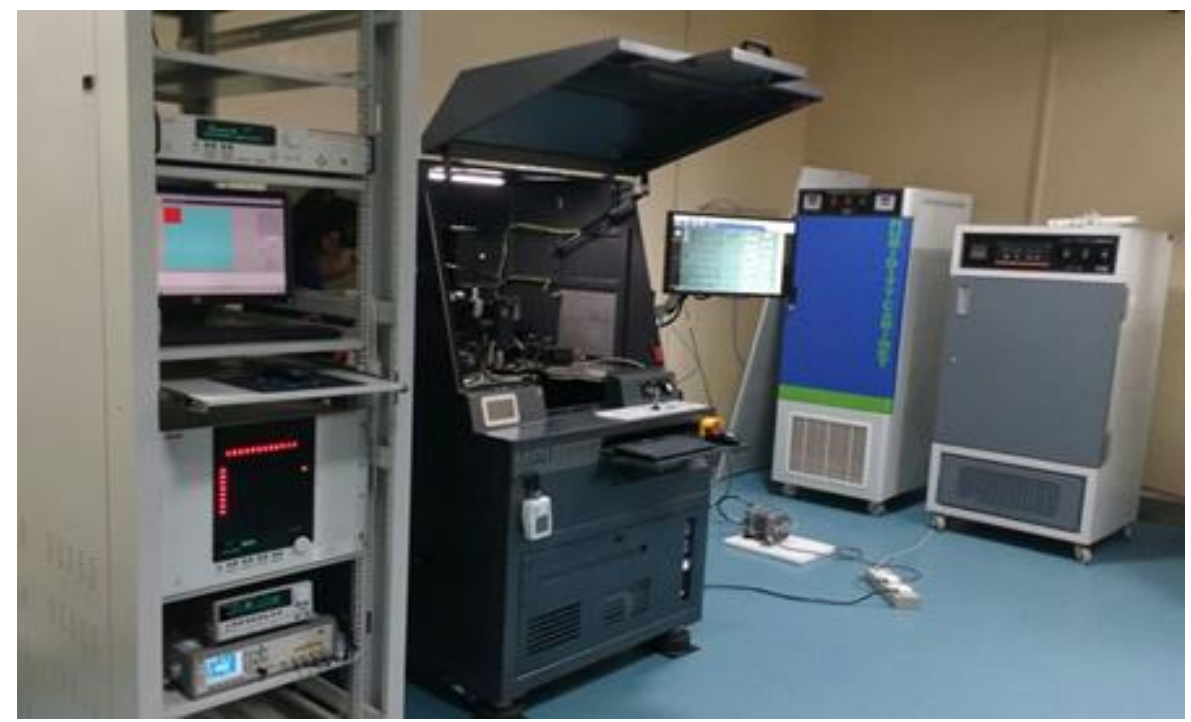

Figure 1 : Characterization system along with the set of electrical instruments at DU.

\section{Description of the sensor and parameters to be tested}

In the present work we have characterized multi micro-strip silicon sensors, fabricated by an Indian foundry, Bharat Electronics Limited (BEL), Bengaluru, India.

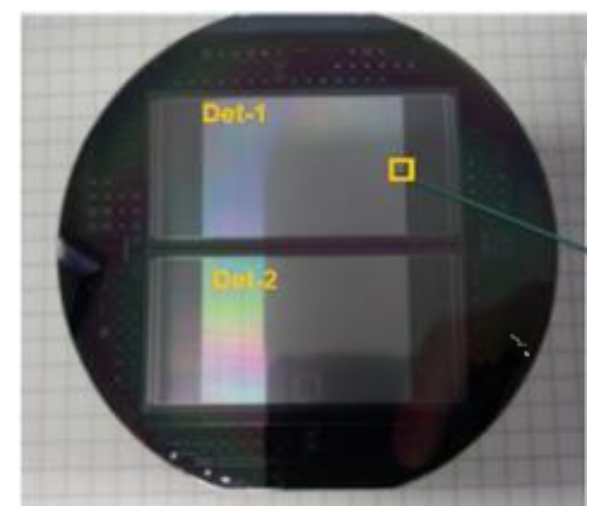

Figure 2 : BEL fabricated silicon multi micro-strip sensor. 
These sensors are AC-coupled, p-on-n, poly-silicon biased, developed on a 4-inch wafer of $300 \mu \mathrm{m}$ thickness. These sensors have a total of 512 strips, each of width $30 \mu \mathrm{m}$ and pitch 55 $\mu \mathrm{m}$. Figure 2 shows a photograph of one such 4-inch wafer with 2 sensors and various test structures on the periphery fabricated on it. Detailed description of this sensor can be found in Ref. [3, 4]. These sensors are characterized for eight different parameters, categorized as global, strip and inter-strip as described below.

Global parameters : Total leakage current (Itot), backplane capacitance (Ctot)

Strip parameters : Strip leakage current (Istrip), Poly-silicon bias resistance (Rpoly), Dielectric current (Idiel), Coupling capacitance (Ccoup)

Inter-strip parameters : Inter-strip Resistance (Rint), Inter-strip Capacitance (Cint)

We also need to keep track of the environmental conditions which include temperature and relative humidity $(\mathrm{RH})$ inside the probe station chamber.

\section{Measurement Results}

Figure 3 shows the results of all the eight measurements on one such sensor. The strip and inter-strip parameters are measured on alternate strips of the sensor.

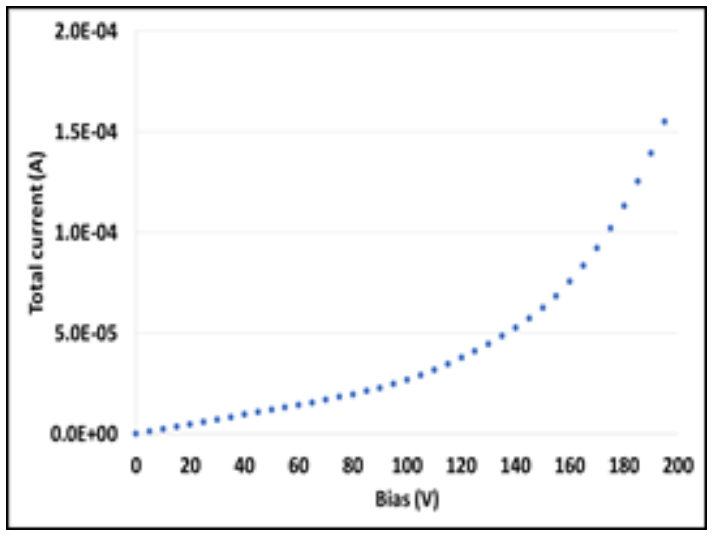

(a)

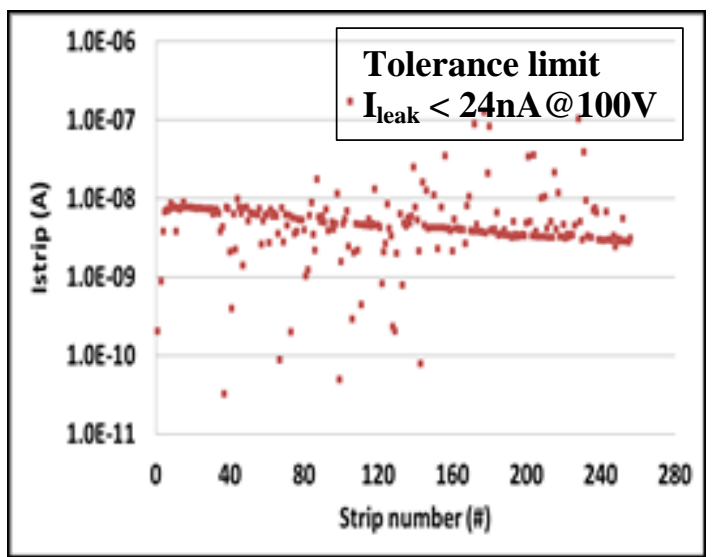

(c)

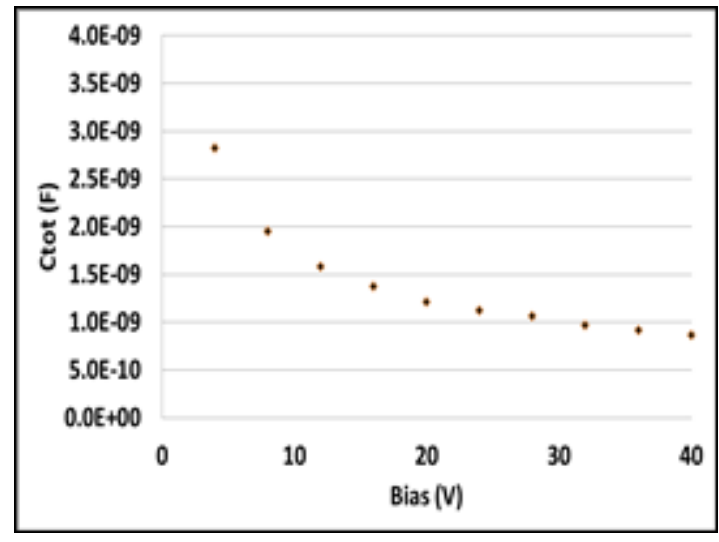

(b)

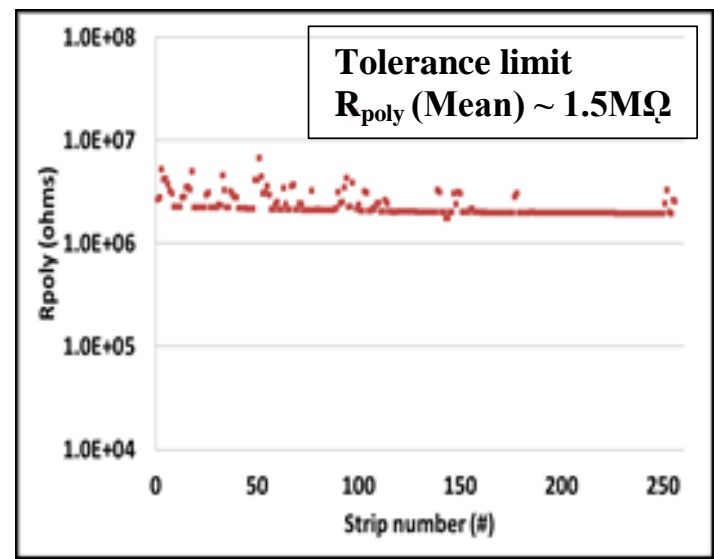

(d) 


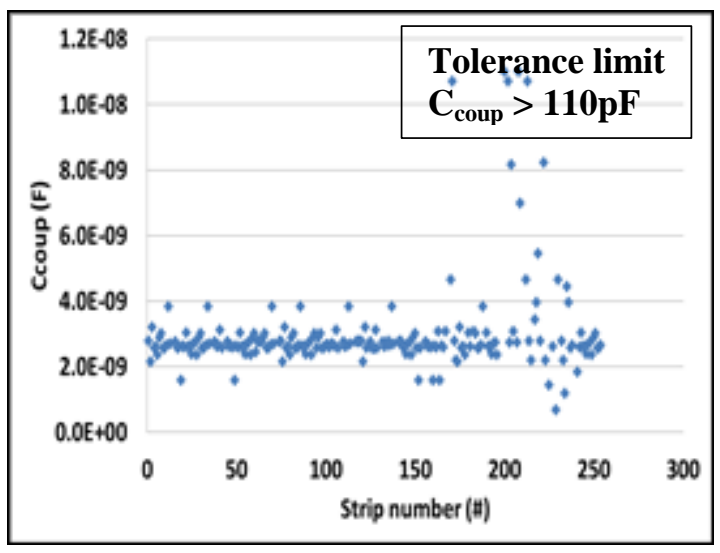

(e)

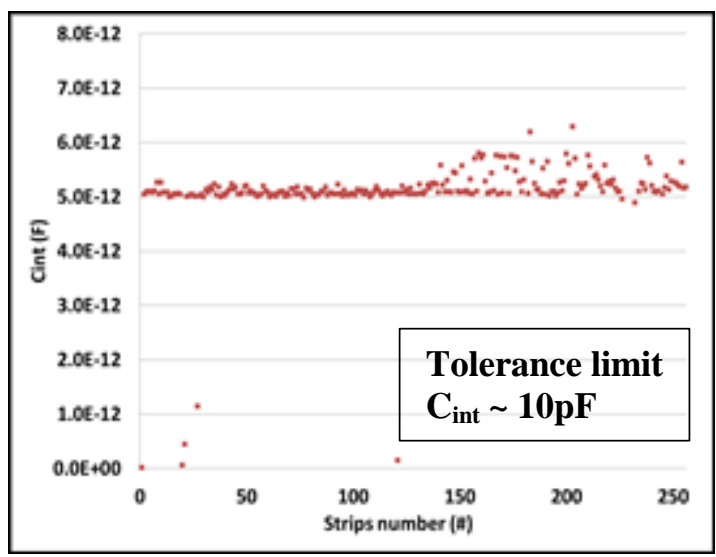

(g)

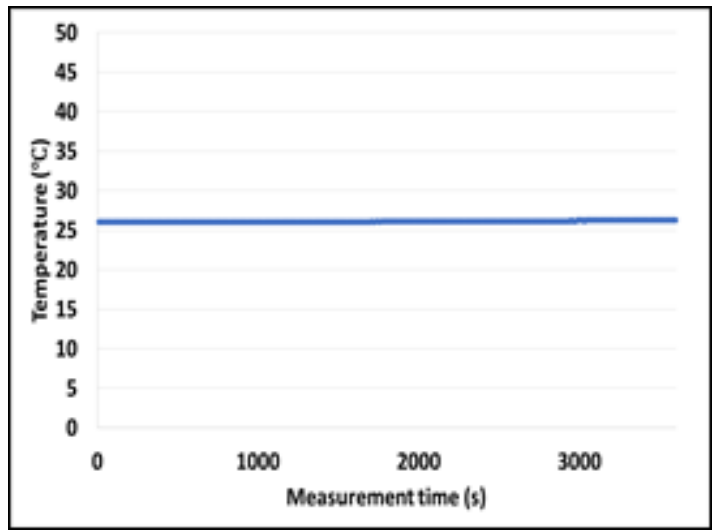

(i)

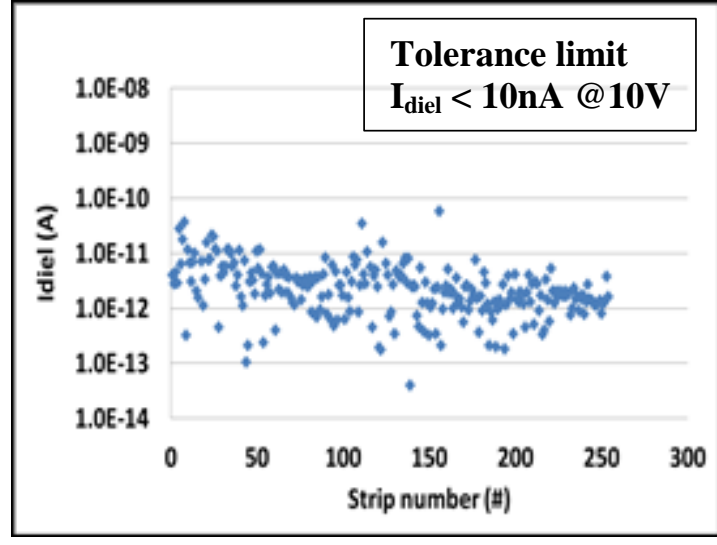

(f)

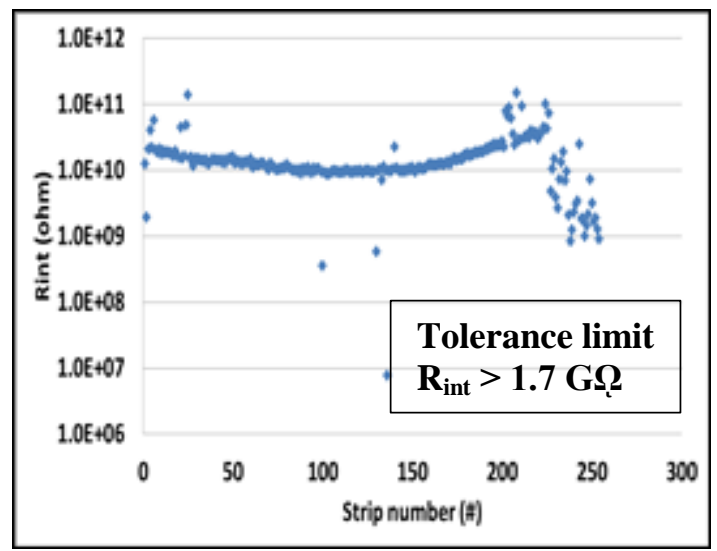

(h)

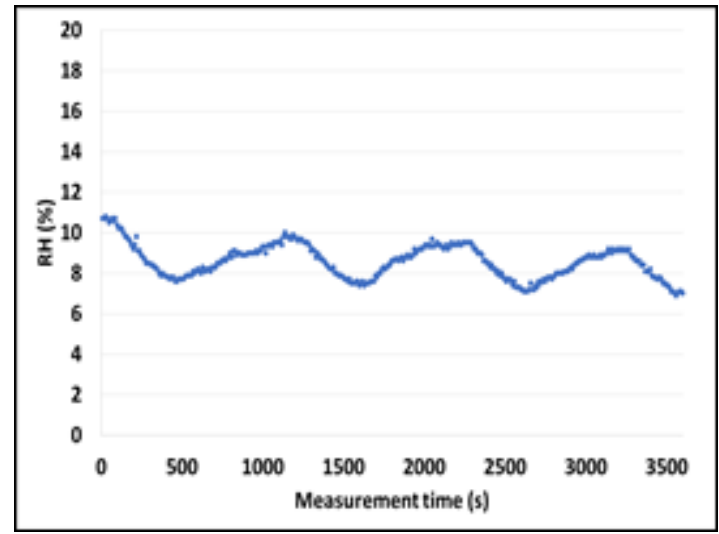

(j)

Figure 3 : Measurement results of all the 8 parameters taken at DU. Voltage scan of (a) total leakage current, (b) backplane capacitance, strip scan of (c) strip current, (d) poly-silicon bias resistor, (e) Coupling capacitance, (f) dielectric current, (g) inter-strip capacitance and (h) inter-strip resistance and time scan of environmental conditions (i) temperature and (j) relative humidity. 
The plot in Fig 3(a) shows the total leakage current which is of the order of $\mu \mathrm{A}$ up to 100 $\mathrm{V}$. However, there is an early onset of soft breakdown around 120V. The strip scans (Figures 3(c) to 3(h)) of various strip and inter-strip parameters are measured at a bias voltage of $200 \mathrm{~V}$ and show reasonable homogeneity over the strips. Figure 3(c) shows that the strip leakage current remains well below $8 \mathrm{nA}$ at $200 \mathrm{~V}$. Figure 3(d) shows that the value of Rpoly remains more than $2 \mathrm{M} \Omega$ for almost all the strips. It can be seen from Figure 3(e) that the value of Ccoup remains higher than $2 \mathrm{nF}$ for most of the strips which ensures a good coupling of the signal to the read-out strips. Figure 3(f) shows that the value of Idiel remains lower than $10 \mathrm{pA}$ at $10 \mathrm{~V}$ which shows a good quality of coupling oxide. Figures $3(\mathrm{~g})$ and $3(\mathrm{~h})$ show the values of interstrip parameters Cint and Rint that remain lower than $6 \mathrm{pF}$ and more than $8 \mathrm{G} \Omega$ respectively, which ensures a good isolation between the neighboring strips. The repeatability of the measurements is also observed in the measurements. Also, we can see a good stability of temperature and relative humidity during the measurements.

\section{Summary}

The silicon sensor automated characterization facility is developed and measurements are performed on strip sensor fabricated at BEL, Bengaluru. This facility is proposed to be used for characterization and qualification of the $2 \mathrm{~S}$ sensors during the production phase of the CMS Outer Tracker Phase-II Upgrade in HL-LHC phase.

\section{Acknowledgments}

The authors acknowledge the financial support from the DST, UGC and the University of Delhi.

\section{References}

[1] HL-LHC home page. http://hilumilhc.web.cern.ch/

[2] CMS Collaboration, The Phase-2 Upgrade of the CMS Tracker Technical Design Report. CERN LHCC-2017-009, CMS-TDR-17-001 (2017).

[3] G. Jain, et al., Development of AC-coupled, poly-silicon biased, p-on-n Si strip detectors in India for HEP experiments, NIM A 882 (2018) 1-10.

[4] G. Jain, et al., Radiation Tolerance Study on Irradiated AC-coupled, Poly-silicon biased, p-on- $n$ Silicon Strip Sensors developed in India, NIM A 913 (2019) 97-102. 